\title{
FACTIBILIDAD DE IMPLEMENTAR PRONOSTICADORES AUTOMATIZADOS PARA CONTROLAR EL TIZÓN TARDIO DE LA PAPA EN EL SUR DE CHILE
}

\author{
Pilar Flores N. ${ }^{1}$, Juan Lerdon F. ${ }^{1}$, Rodrigo Bravo H. ${ }^{2}$, e Ivette Acuña ${ }^{2}$ \\ ${ }^{1}$ Universidad Austral de Chile, Facultad de Ciencias Agrarias, Instituto de Economía Agraria. Casilla \\ 567, Valdivia, Chile. Jlerdon.@uach.cl; ${ }^{2}$ Instituto de Investigaciones Agropecuarias, Centro \\ Regional de Investigación Remehue, Casilla 24-O, Osorno, Chile. rbravo@inia.cl.
}

\section{ABSTRACT \\ Feasibility of implementing an automated forecasting system to control potato late blight in Southern Chile.}

Key words: Forecasting disease system, late blight, potato

An analysis of the potato yield from experimental plots established in the 2004-2005 season in potato growing areas of the IX and X region was carried out, with 4 phytosanitary treatments compared in each experimental site. An automated alarm system to detect potato late blight disease was analyzed, from climatic data compiled and later processed through the ISO 9000 Standard. The financial economical analysis that was conducted included estimations of the following indices: Net Present Value (NPV), Internal Rate of Return (IRR) and breakeven point. The system appears to be a viable alternative, with values of $\$ 1$ and $12 \%$ for NPV and IRR, respectively.

\section{RESUMEN}

Palabras claves: Sistema de pronósticos de enfermedad, tizón tardío, papa.

Se realizó un análisis del rendimiento obtenido en parcelas experimentales establecidas en la temporada 2004-2005, en zonas de importancia como productoras de papas de la IX y X región, donde se compararon en cada sitio experimental 4 tratamientos.

Se analizó el funcionamiento del sistema de alarma del tizón tardío de la papa, desde la recopilación de datos climáticos hasta su posterior procesamiento a través de la Normativa ISO 9000.

El análisis económico financiero se basó en el cálculo de los indicadores: valor actual de los beneficios netos (VABN), tasa interna de retorno (TIR) y punto de equilibrio.

Los resultados obtenidos permiten señalar que el proyecto desarrollado representa una alternativa viable, obteniendo valores de $\$ 1$ y $12 \%$ de VABN y TIR respectivamente.

\section{INTRODUCCION}

La papa (Solanum tuberosum L.) es uno de los cuatro alimentos básicos más importantes de la humanidad, ocupando una superficie de aproximadamente 18 millones de hectáreas, con una producción cercana a 300 millones de toneladas, de las cuales cerca de 170 millones son destinadas al consumo humano (Fundación Chile, 2001).
En Chile, este cultivo es de gran importancia tanto social como económica, ocupando el tercer lugar en superficie entre los cultivos anuales, el segundo lugar en cuanto al valor de su producción y el primer lugar en el valor de las jornadas/ hombre requeridas en su explotación (Santos et al., 2001). Además, es cultivada por distintos tipos de agricultores, diferenciándose en su nivel tecnológico, número de hectáreas y en la zona de producción (Bravo y Holmberg 2003). 
Una de las grandes limitantes en la producción de papa son los problemas fitopatológicos, los que afectan tanto el rendimiento como la calidad del producto (Acuña, 2004). Dentro de las enfermedades, el tizón tardío causado por el hongo Phytophthora infestans, es una de las más importantes en el cultivo de la papa a nivel mundial (ICOCHEA, 1980, Fernández, 1994, Acuña y Torres, 2000). Las condiciones climáticas de la zona sur de Chile varían cada año, lo que ha llevado a una inseguridad en el manejo de esta enfermedad, en cuanto a la oportunidad de aplicación de fungicidas, teniendo como consecuencia el control inadecuado o la falta de este y un exceso en el uso de pesticidas (INIA, 2003).

Dado lo anterior, se considera como hipótesis del estudio, que es factible y económicamente viable, implementar pronosticadores automatizados para controlar el tizón tardío de la papa, en los casos estudiados.

Su objetivo general es evaluar el uso de pronosticadores para controlar el tizón tardío en el cultivo de la papa, para agricultores de las regiones IX y X de Chile, logrando así un uso eficiente y racional de los fungicidas disponibles para su control.

Los objetivos específicos son: Describir un sistema de alerta temprana para detectar presencia de tizón tardío en el cultivo de la papa; estimar los beneficios económicos en la producción de papa con un sistema de alerta temprana para el tizón tardío y determinar la factibilidad económica del servicio de alerta temprana para dicha enfermedad.

\section{MATERIAL Y METODO}

Información de parcelas experimentales. Se utilizaron datos de rendimiento obtenidos de las parcelas experimentales, que fueron establecidas para determinar la calibración y efectividad del sistema de control. Estas parcelas se establecieron en la temporada 2005-2006, en zonas paperas importantes de la IX y X Región, donde se compararon en cada lugar 4 tratamientos, siendo el T1: el testigo; sin aplicación de productos fungicidas para el control del tizón tardío; T2: aplicación a calendario fijo; T3: aplicación de acuerdo a la alarma DACOM y el T4: tratamiento calendario fijo alternativo. En el Cuadro 1 se observa el lugar donde se estableció cada estación meteorológica, existiendo solo 2 estaciones conectadas en la red, razón por la cual se extrapolaron los pronósticos de estas estaciones para las otras localidades, pudiendo así estimar la alarma para el tratamiento 3 .

Procesos tecnológicos. Se recurrió a seminarios, libros y entrevistas con especialistas en el tema para analizar aspectos técnicos y económicos del servicio de alerta temprana.

Análisis estadístico del rendimiento de las parcelas experimentales. Los resultados obtenidos de las parcelas experimentales sirvieron como información para medir diferencias significativas de cada alternativa o estrategia, a través de regresiones múltiples mediante el software Statistical Analysis System (SAS). Las diferencias entre tratamientos y lugares fueron cuantificadas mediante

Cuadro 1. Ubicación de las estaciones meteorológicas en la IX y X región.

Table 1. Location of the weather stations in the IX and $\mathrm{X}$ regions.

\begin{tabular}{|c|c|c|c|}
\hline Región & Localidad & Propietario & Ubicación \\
\hline \multirow[t]{4}{*}{ IX } & Carillanca & INIA & Lat. $38^{\circ} 41^{\prime} \mathrm{S} /$ Long. $72^{\circ} 25^{\prime} \mathrm{W}$ \\
\hline & Teodoro Schmidt & Liceo Cristo Rey & Lat. $38^{\circ} 59^{\prime} \mathrm{S} /$ Long. $73^{\circ} 05^{\prime} \mathrm{W}$ \\
\hline & Tranapuente & INIA & Lat. $38^{\circ} 41^{\prime} \mathrm{S} /$ Long. $73^{\circ} 21^{\prime} \mathrm{W}$ \\
\hline & Lago Budi & U. Católica de Temuco & Lat. $38^{\circ} 52^{\prime} \mathrm{S} /$ Long. $73^{\circ} 14^{\prime} \mathrm{W}$ \\
\hline \multirow[t]{3}{*}{$\mathrm{X}$} & La Pampa & INIA & Lat. $40^{\circ} 52^{\prime} \mathrm{S} /$ Long. $73^{\circ} 12^{\prime} \mathrm{W}$ \\
\hline & Los Muermos & FIA & Lat. $41^{\circ} 28^{\prime} \mathrm{S} /$ Long. $73^{\circ} 28^{\prime} \mathrm{W}$ \\
\hline & Butalcura & FIA & Lat. $42^{\circ} 15^{\prime} \mathrm{S} /$ Long. $73^{\circ} 39^{\prime} \mathrm{W}$ \\
\hline Servidor & Remehue & FIA & Lat. $40^{\circ} 35^{\prime} \mathrm{S} / \mathrm{Long} .73^{\circ} 08^{\prime} \mathrm{W}$ \\
\hline
\end{tabular}


comparación de promedios mínimos cuadráticos, aceptándose una probabilidad de significancia del $5 \%$.

Cuantificación económica del rendimiento de las parcelas experimentales. El rendimiento obtenido de las parcelas experimentales sirvió para analizar en cada sector el costo/rendimiento incremental de cada tratamiento para determinar cual de ellos era económicamente factible de utilizar.

Procesos tecnológicos. Para el análisis del funcionamiento del sistema de alarma del tizón tardío de la papa, se utilizó la Normativa ISO 9000 , de tal forma de obtener una mayor eficiencia desde la recopilación de datos climáticos hasta su posterior procesamiento, para así generar recomendaciones a los usuarios en el control de esta enfermedad.

Estudio económico. Se estableció la estructura de la inversión, los costos operacionales e ingresos brutos para confeccionar el flujo de caja anual.

Estudio financiero. El análisis financiero consideró la determinación de los siguientes índices: valor actual de los beneficios netos (VABN), tasa interna de retorno (TIR) y punto de equilibrio, con 10 estaciones meteorológicas. El horizonte de evaluación del proyecto fue de diez años, considerando la vida útil del principal elemento de la estación, el datalogger, que es la unidad encargada de monitorear en forma sistemática los sensores de la estación meteorológica.

\section{PRESENTACION Y DISCUSION DE RESULTADOS}

Resultados de alerta temprana para detectar el tizón tardio a través del modelo computacional. En el Cuadro 2 se indican las fechas de alarma para aplicar productos fungicidas para el tratamiento 3 , en las distintas localidades donde se ubicaban las parcelas experimentales. De esta forma, queda demostrado que es posible dar aviso e informar a los agricultores sobre esta enfermedad, donde ha sido reportada y generar su posterior recomendación, de acuerdo a la intensidad del ataque del hongo al cultivo. Se excluyeron de este análisis las parcelas situadas en el Lago Budi, Tranapuente y Butalcura, ya que hubo problemas de comunicación de la alarma del tizón tardío en dichos lugares, lo que generó error en las mediciones.

Resultados estadísticos del rendimiento de las parcelas experimentales. En los Cuadros 3 y 4 , se muestran los resultados de rendimiento comercial, desecho y total de estos, obtenidos a través de las medias mínimo cuadráticas de los cuatro tratamientos y del lugar, respectivamente. Con los resultados del Cuadro 3, se puede inferir que no existe diferencia significativa entre los tratamientos T2, T3 y T4, pero sí con el tratamiento testigo (T1). Se puede decir, entonces, que cualquiera de los tratamientos realizados tuvo el mismo efecto de control en la temporada analizada, entonces, un sistema de alerta temprana, puede ser más eficiente que un sistema de control a calendario fijo, pudiendo reducir el número de aplicaciones realizadas. En cuanto al análisis por sector (Cuadro 4), se obtuvo diferencias significativas en todos los lugares.

Resultados de los costos de cada tratamiento. En este ítem no se tomó en cuenta el tratamiento testigo, al no tener costos de aplicación de

Cuadro 2. Fechas de alarma de tizón tardío en las distintas localidades de la IX y X región. Temporada 2004-2005. Table 2. Dates of late blight alarms in the different locations from the IX and X regions during the 2004-2005 season.

\begin{tabular}{llllll|}
\hline Localidad & \multicolumn{5}{c|}{ Fechas de alarma del tizón tardío } \\
\hline INIA-Remehue & 18-Ene & 07-Feb & 14-Feb & 25-Feb & 03-Mar \\
INIA-La Pampa & 10-Ene & 21-Ene & 31-Ene & 10-Feb & 21-Feb \\
Los Muermos & 10-Ene & 21-Ene & 31-Ene & 10-Feb & 21-Feb \\
INIA-Carillanca & 24-Ene & 07-Feb & 16-Feb & 02-Mar & 07-Mar \\
T. Schmidt & 22-Dic & 08-Ene & 03-Feb & & \\
\hline
\end{tabular}


Cuadro 3. Medias mínimo cuadráticas de los cuatro tratamientos.

Table 3. Average minimum quadratic of the four treatments.

\begin{tabular}{|l|rrrr|}
\hline \multicolumn{1}{|c|}{$\begin{array}{c}\text { Rendimiento } \\
\text { (t/ha) }\end{array}$} & \multicolumn{5}{|c|}{ Tratamiento } \\
\cline { 2 - 5 } & T1 & \multicolumn{5}{|c|}{ T2 } & T3 & T4 \\
\hline Comercial & $29,66 \mathrm{~b}$ & $39,40 \mathrm{a}$ & $39,37 \mathrm{a}$ & $42,11 \mathrm{a}$ \\
Desecho & $3,94 \mathrm{a}$ & $2,41 \mathrm{~b}$ & $2,56 \mathrm{~b}$ & $2,16 \mathrm{~b}$ \\
Total & $33,60 \mathrm{~b}$ & $41,81 \mathrm{a}$ & $41,92 \mathrm{a}$ & $44,27 \mathrm{a}$ \\
\hline
\end{tabular}

Los valores de cada línea horizontal seguidos con distinta letra indica diferencia estadísticamente significativas $(\mathrm{P}<0,05)$

Cuadro 4. Medias mínimo cuadráticas de los sectores IX y X región.

Table 4. Average minimum quadratic of sectors in the IX and $X$ regions.

\begin{tabular}{|l|cccccc|}
\hline \multirow{2}{*}{$\begin{array}{c}\text { Rendimiento } \\
\text { (t/ha) }\end{array}$} & \multicolumn{5}{|c|}{ Lugar } \\
\cline { 2 - 6 } & Remehue & La Pampa & Los Muermos & T. Schmidt & Carillanca \\
\hline Comercial & $57,22 \mathrm{a}$ & $49,59 \mathrm{~b}$ & $37,60 \mathrm{c}$ & $25,71 \mathrm{~d}$ & $18,03 \mathrm{e}$ \\
Desecho & $2,54 \mathrm{c}$ & $4,07 \mathrm{~b}$ & $1,65 \mathrm{~d}$ & $4,93 \mathrm{a}$ & $0,64 \mathrm{e}$ \\
Total & $59,76 \mathrm{a}$ & $53,66 \mathrm{~b}$ & $39,26 \mathrm{c}$ & $30,64 \mathrm{~d}$ & $18,67 \mathrm{e}$ \\
\hline
\end{tabular}

Los valores de cada línea horizontal seguidos con distinta letra indica diferencia estadísticamente significativas $(\mathrm{P}<0,05)$

productos fungicidas. En el Cuadro 5 se puede observar que en los sectores Carillanca, Teodoro Schmidt y Los Muermos el tratamiento más económico lo representa el T4, o aplicación fija alternativa, donde en el sector Carillanca presenta una diferencia de $\$ 11.990$ con el T2 y $\$ 31.854$ con el T3. En el sector Teodoro Schmidt, esta diferencia no es tan significativa como en el sector anterior, existiendo una diferencia de $\$ 4.919$ con el T2 y de $\$ 935$ con el T3. Finalmente, en el sector Los Muermos, la diferencia fue de $\$ 3.553$ con el T2 y $\$ 13.617$ con el T3. En cuanto a los sectores Remehue y La Pampa, se puede apreciar que el costo/ rendimiento adicional de los tres tratamientos es muy similar, no pudiéndose hacer diferencias entre ellos y determinar cual es más económico.

De esta manera, se consideró un valor máximo de $\$ 15.801$ con un número de agricultores de 814 , según el Cuadro 6. Un precio mayor de cobro del servicio, sería un valor muy alto, ya que hay que considerar que el agricultor podría hacer más aplicaciones de productos fungicidas para controlar el tizón tardío.

Estudio financiero del proyecto. En el Cuadro 7 se detalla la inversión del proyecto y en el Cuadro 8 se presentan los costos anuales que se incurrieron durante el estudio.

Cuadro 5. Costo/rendimiento adicional de cada tratamiento en las diferentes localidades de las regiones IX y X. Table 5. Cost/additional yield of each treatment in the different localities from the IX and $\mathrm{X}$ regions.

\begin{tabular}{|c|ccccc|}
\hline & Carillanca & T. Schmidt & Remehue & La Pampa & Los Muermos \\
\hline T2 & 31,665 & 9,624 & 7,384 & 14,246 & 12,882 \\
T3 & 51,529 & 5,640 & 7,367 & 13,981 & 22,946 \\
T4 & 19,675 & 4,705 & 7,399 & 14,308 & 9,329 \\
\hline
\end{tabular}


Cuadro 6. Precio del servicio según el número de agricultores.

Table 6. Price of the service according to the number of farmers.

\begin{tabular}{|c|c|}
\hline Precio del servicio (\$) & $\mathrm{N}^{\mathrm{o}}$ de agricultores \\
\hline 3.950 & 3.255 \\
7.900 & 1.628 \\
15.801 & 814 \\
31.601 & 407 \\
63.203 & 203 \\
126.406 & 102 \\
252.812 & 51 \\
505.623 & 25 \\
1.011 .246 & 13 \\
\hline
\end{tabular}

Cuadro 7. Activos fijos del servicio de alerta temprana.

Table 7. Fixed assets of the early alarm service.

\begin{tabular}{|l|c|c|c|}
\hline Equipamiento & Costo unitario (\$) & Cantidad & Costo Total(\$) \\
\hline Modulo de comunicación & 139.496 & 10 & 1.394 .958 \\
PC genérico Servidor & 504.201 & 1 & 504.201 \\
Batería & 30.000 & 10 & 300.000 \\
Estaciones metereológicas & & & \\
DL 2 & 2.992 .015 & 2 & 5.984 .030 \\
Hog & 2.544 .567 & 1 & 2.544 .567 \\
HL-20 & 2.400 .000 & 3 & 7.200 .000 \\
Vantage Pro2 & 1.550 .000 & 1 & 1.550 .000 \\
CR10X & 3.805 .799 & 3 & 11.417 .397 \\
Software pronosticador & 1.500 .000 & 1 & 1.500 .000 \\
Cerco para estaciones & 13.414 & 10 & 134.140 \\
\hline Total inversión & & & 32.529 .293 \\
\hline
\end{tabular}

Se consideró para la evaluación una tasa de descuento de un $12 \%$, incluida una tasa de riesgo. El horizonte de evaluación fue de 10 años. El valor de desecho, correspondió al monto de la inversión en activos fijos al final del proyecto, más las reinversiones y las nuevas inversiones en activos fijos realizadas en el $5^{\circ}$ año debidamente depreciadas, siendo su valor contable de \$4.687.729.

Rentabilidad del proyecto. Se consideró un perfil del proyecto de 10 años y los índices económicos calculados fueron: El valor actual de los beneficios netos (VAN) dando como resultado $\$ 3$, la tasa interna de retorno (TIR) con un valor de $12 \%$ y el punto de equilibrio para el tercer año del proyecto, con un valor de $29 \%$. De acuerdo a estos resultados, el proyecto sería viable y rentaría lo que el inversionista exige a la inversión. En efecto, el criterio del VAN establece que el resultado debe ser igual o superior a cero y según el criterio de laTIR el resultado debe ser igual o mayor a la tasa de descuento (Lerdon, 2004).

Cuadro 8. Costos anuales.

Table 8. Annual costs.

\begin{tabular}{|l|c|}
\hline Item & Monto (\$) \\
\hline Costos fijos & 3.730 .234 \\
Costos variables & 2.275 .212 \\
\hline Total (\$) & 6.005 .446 \\
\hline
\end{tabular}




\section{CONCLUSIONES}

- Se acepta la hipótesis, en el sentido que es factible y económicamente viable implementar los pronosticadores automatizados para controlar el tizón tardío de la papa, en los casos estudiados. Si bien los resultados obtenidos podrían haber sido mejores, esta experiencia aporta los primeros antecedentes de una nueva tecnología que se quiere implementar, para controlar el tizón tardío de la papa y que a futuro, se pretende mejorar con la experiencia adquirida en este proyecto.

- Según la Normativa ISO 9000, este proyecto cumple con los requisitos exigidos, por lo que es aceptado como una alternativa de control de esta enfermedad.

- Se puede inferir que cualquier tratamiento de productos fungicidas que se utilice, se va a traducir en un mayor rendimiento, en comparación a no hacer ningún control. En cuanto al análisis por sector, se obtuvo mayor rendimiento comercial y total en el sector INIARemehue y el mayor rendimiento desecho en el sector Teodoro Schmidt.

- En cuanto a los beneficios económicos del sistema de alerta temprana, se estimó que el sector Carillanca, Teodoro Schmidt y Los Muermos, el tratamiento más económico lo representa el T4. En los sectores INIA-Remehue e INIA-La Pampa, el costo/rendimiento adicional de los tres tratamientos son similares, no pudiéndose hacer diferencias entre ellos, para determinar el más económico.

- De acuerdo a los resultados de los indicadores de rentabilidad utilizados, el proyecto se presenta como una alternativa viable para un número de 814 agricultores.

\section{BIBLIOGRAFIA}

ACUÑA, I. y TORRES, H. 2000. El Tizón tardío de la papa. Osorno, Chile. Boletín $\mathrm{N}^{\circ} 22.2$ p.

ACUÑA, I. 2004. Cómo reconocer los tizones de la papa. Osorno, Chile. Boletín $N^{\circ} 47.2$ p.

CHILE, MINISTERIO DE AGRICULTURA, INSTITUTO DE INVESTIGACIONES AGROPECUARIAS (INIA). 2003. In: Proyecto Uso de pronosticadores para el desarrollo de estrategias de manejo del Tizón tardío de la papa en la zona sur de Chile. INIA Remehue. Osorno, Diciembre. 118 p.

BRAVO, R. y HOLMBERG, G. 2003. Perspectivas y Desafíos tecnológicos del rubro papa. In: Seminario Avances en Producción de Papa en la IX Región. INIA Carillanca. Temuco, 3 de Septiembre. pp: 45-52.

FERNÁNDEZ, C. 1994. Enfermedades fungosas de la papa: Importancia, detección y control. Tizón tardío de la papa (Phytophthora infestans). In Rojas, J., Accatino, P. y Kalazich, J. (eds). Metodología para mejorar la producción y uso de tubérculos-semillas de papa en Chile. Serie Remehue $\mathrm{N}^{\circ}$ 51. INIA. pp. 89-98.

FUNDACIÓN CHILE. 2001. Cadenas agroalimentaria, Papas. (On line). <http:// www.funch.cl/fc/papas > (8 abr. 2005).

ICOCHEA, T. 1980. Tizón tardío de la papa. In Hooker, W. (ed). Compendio de enfermedades de la papa. Centro Internacional de la Papa. Lima. Perú. pp: 56-60.

LERDON, J. 2004. Formulación y evaluación de proyectos agrícolas. Apuntes para el curso de Formulación y Evaluación de Proyectos Agrícolas. Instituto de Economía Agraria. Universidad Austral de Chile. Valdivia. 146 p.

SANTOS, J., KALAZICH, J. Y SIERRA, C. 2001.Cultivos industriales. Papa. In: Agenda del salitre. SOQUIMICH. $11^{\circ}$ ed. Santiago, Chile. pp: 657-672. 